\section{đIhe lettramian}

$$
\text { or }
$$

\section{ASEPTIC SURGERY IN THEORY AND PRACTICE.}

Delivered on February. 29th, 1904.

BY CHARLES BARRETT LOCKWOOD, F.R.C.S.ENG., Surgeon, St. Bartholomew's Hospital.

LECTURB III.

On the Accurate Closure of Wounds.-Skin Flaps.Skin Sliding.- Environment and Results.

WhEN the cut edges of the skin are brought together with perfect accuracy, they adhere at once and are an effectual barrier against air infection. In respect to this rapid adherence the skin is comparable to the peritoneum. But to secure this advantage the apposition must be perfect and exact. This point is well understood, but perhaps a word may be said with regard to another aid to asepsis which will, in the future, have an extensive use. I refer to the complete and accurate closure of the largest wounds by the transposition of flaps of skin, by undercutting, and by the gliding of extensive portions at the boundaries of the wound.

Many of us can remember the time when an amputation wound was sewn together with discontinuous sutures about an inch apart; and can recall the little area of raw surface which was left between the sutures. Nowadays this would be considered very poor workmanship. A number of discontinuous sutures might be used to adjust the flaps and obliterate the space between them, but every vestige of the raw surface would now be covered in by running a continuous suture of the finest twisted silk along the whole of the edges of the wound. The skin adheres with great rapidity, and is probably firmly gunited before any bacteria which may have survived in the sweat glands, sebaceous glands, or hair follicles could have had time to multiply and invade the deeper parts of the wound. We know how rare a perfect disinfection of the skin must be. When we come to consider the matter, it would but a short time ago have been rather rash to have aimed at such accurate apposition, because then, in all probability, it would have led to the retention of septic fluids within the wound, and to such a series of disasters as can be occasionally found recorded in the older works on surgery.

In many operations, especially in those for mammary carcinoma, a very large area of skin has to be removed, as it is now recognized that the skin over the cancerous growth cannot be saved with safety because its lymphatics are frequently the seat of cancerous infection.

The excision of the suspected skin often leaves a huge gap which can only be closed by the exercise of considerable ingenuity. When the wound is aseptic, flaps of skin and subcutaneous tissue, six or seven inches long and attached by quite a narrow base, can be dissected up and turned into the wound. These flaps hardly possess proper blood supply of their own and only survive because, owing to the asepticity of the wound, they immediately adhere to the chest wall to which they are applied. From that source they do not at first acquire new blood vessels, but are probably kept alive by the absorption of plasma. It is remarkable to observe how tight these huge skin flaps may be, and how livid they may become, and yet preserve their vitality. At the end of a few days they may appear to be upon the point of perishing, but nevertheless recover and unite firmly with all their surroundings. I have used similar skin flaps to close gaps in the chest wall. About eighteen months ago in removing recurrent carcinoma portions of three ribs and a large piece of pleura had to be excised. A large area of the diaphragm and of the lung was exposed to view. Four or five sponges were stuffed into the gap, whilst a large thick flap was brought up from the chest and abdomen. The patient recovered withou a single bad symptom. Without the aid of the flap this operation could not have been undertaken; and without asepsis the flap could not have been made.

The principles which govern the fashioning of these flaps cannot be entered upon now. The theory is simple, the praccice difficult. I shall content myself with saying that there is room for a whole lecture upon this subject, which, by the bye, has been almost entirely neglected by surgical writers.

For the complete closure of gaping wounds asepsis allows us to use a further device. Besides being able to convert the surrounding skin into flaps, we can also undercut it so as to allow large pieces to slide in the direction of the wound Doubtless this undercutting destroys in part the blood and nerve supply of the skin, but so long as the wound is aseptic no harm results.

In bringing together the wound and adjusting the edges of skin flaps a continuous suture of twisted silk is used. This has one great advantage after a protracted operation such as that for removal of the mammary gland, pectoral muscles, and the lymphatic area-it is very rapid. But the continuous suture has a disadvantage for it often strangulates the strip of skin within its grasp. As a rule, when this occurs the loss is trifling, but it is to be doubted whether the wound ever remains aseptic, and at times the ultimate healing is delayed. For this reason I have found it difficult to classify as "septic" or "aseptic" some of the amputations of the breast and operations for cancer of the lymphatics of the neck. Without an explanation it would lead to misconception if they were called septic, and yet they cannot truthiully be called aseptic. Some day, when our knowledge is more precise, we shall perhape include them in a class labelled " "secondary skin infection after necrosis of skin".

In spite of these occasional drawbacks, the complete closure of wounds by means of skin sliding and skin flaps is an enormous gain, and, when boldly resorted to, does away with the necessity for skin grafting by Thiersch's or other methods. It is a very great happiness to patients to be told, on the removal of the dressing. that the wound is entirely healed, and they seem to think less of scars which are all in one place.

Drainage.

Some years ago I remember reading a paper before this Society entitled The Abuse of Drainage of Wounds. ${ }^{1}$ About that time (1890) nearly every wound was drained. It is not unlikely but that the pendulum afterwards swung too far in the other direction. I suppose that to-day all septic wounds are drained with gauze, or tubes, or some other efficient device; also all wounds in which blood is liable to collect. I myself always drain wounds of the scrotum, and nearly always those of the axilla, merely to prevent blood collecting. Rubber tubes are the most comfortable for the patient, and can be sterilized with heat. Glass tubes are apt to hurt, and on more than one occasion have been known to break within the abdomen, but nevertheless they are so efficient and so easily sterilized that I occasionally employ them; gauze has advantages, but is painful to remove, even with the help of hydrogen peroxide solution. The introduction of a drainage tube increases the risks of infection. Doubtless infection from the skin - which, as we know, is septic in 25 per cent. of cases - can more easily enter when the lips of the wound are kept apart by a drainage tube than when they are allowed to adhere by immediate union. In addition, the wound has to be dressed for the removal of the tube. These considerations have led to the immediate closure of wounds made to empty psoas, lumbar, and iliac abscesses. At the worst, if the operation be aseptic, some pus may collect again and call for removal; but at this stage the abscess cavity is much smaller, and the dangers of sepsis are less. For the successful performance of this operation for emptying and then closing again these large tuberculous abscesses it is of the greatest importance to be gentle and restrained, so as not to start haemorrhage into the abscess cavity. The neglect of this is likely to bring an excellent operation into disrepute.

In aseptic wounds, then, drainage is unnecessary unless oozing and accumulation of blood are anticipated; but in septic wounds drainage is almost invariably required. This bald statement, however, ought to be amplified, for septic cavities ought also to be drained. This is very clear in the case of the urinary bladder. On more than one occasion I have closed the cystotomy wound at once, being guided by the absence of bleeding and of sepsis. It is a vast gain to the patients if the suprapubic wound heals by first intention, and that they should be able to walk about at the end of the third week. But when the bladder is septic, the immediate closure of the cystotomy wound is fraught with danger. Whenever the bladder is septic I insert a Guyon's drainage tube. This admirable device has robbed this class of case of a great many of its dangers and discomforts.

Also, when the bladder is septic I perform external urethrotomy in preference to internal, in order to provide adequate drainage. The dangers of an incised wound far from the surface of the body, and frequently bathed in septic urine, are too obvious to need pointing out. The perineal wound drains well, and merely needs the insertion of a strip of 
gauze. When the urine is aseptic the perineal wound may be closed forthwith, with a good chance of immediate union. The treatment of the gall bladder is ruled by similar principles. If, after cholecystotomy, it were known that the gall bladder was aseptic, and was destined to remain so, it might be judicious to suture the opening into it, and drop it back into the abdomen. But one must always be doubtful about the asepticity of a cavity which communicates with the intestinal tract by a comparatively short canal. No doubt so long as the cystic duct and the common bile duct are undilated and are lined with healthy epithelium, they prevent the intestinal bacteria from invading the gall bladder, the biliary ducts and canals, and the liver substance. But cholecystotomy is usually done when the gall bladder and the ducts have been altered by disease. It is probable that sepsis of the gall bladder is present in every case of gall stones. Acting upon this assumption, I have never yet ventured to suture and return the gall bladder after cholecystotomy, or in other words to perform what has been called the "ideal operation." The systematic drainage of another cavity - the rectum-after the removal of tumours, haemorrhoids, and for operations for fistula, has done much to diminish both the dangers and the miseries of this class of operation. A small ( $\frac{1}{3}$ in.) rubber tube with a packing of iodoform gauze is all that is needed. The gauze supports the drainage tube and stops the bleeding.

To my mind the essential qualities in the dressing are sterility and dryness. Working at St. Bartholomew's and elsewhere with a number of different assistants and sisters, I use plain sterilized gauze, double cyanide gauze, iodoform gauze, and alembroth. For many aseptic cases plain sterilized gauze is now being used. Double cyanide gauze and wool are used for a proportion of aseptic cases, as well as for the septic. For some of the latter iodoform gauze is used. But, as regards the various dressings, I cannot detect any difference in the healing of the wounds provided a layer of silver foil is put next to the skin. If silver foil were not at hand, I should prefer to use plain sterilized gauze and a layer of plain sterilized wool.

This layer of silver foil is supposed to retard the growth of the skin bacteria; next. it helps to exclude air infection; and, lastly, it separates the antiseptic dressing, if such a one be used, from the wound. It is exceedingly hard to trace the line of a wound which has been covered with silver foil because the skin edges are so entirely devoid of redness.

The original Listerian dressing was completed with an outside dressing, which consisted of a layer of waterproof jaconet and eight layers of gauze. The purpose of this outside dressing need not be referred to, but there can be no question but that it has of late fallen into disuse. At some nursing homes I have been told that I am the only surgeon who uses an outside dressing. Now, it may be conceded that the dryness of aseptic wounds has done away with one of the main objects of the outside dressing. Moreover, a large layer of impervious jaconet may be hot and uncomfortable, and, by preventing evaporation, be calculated to favour the collection of perspiration and the growth of skin bacteria. But in some cases these disadvantages are far outweighed by some very solid advantages. For instance, the triangular scrotal dressing which I first described to this Soeiety in $1896^{2}$ is not only comfortable but likewise secure, and since its use a suppurating scrotal wound has been almost unknown. It is true that this result has been attained with the aid of systematic drainage, but that would be risky unless an outside dressing was used to prevent the escaping blood from becoming infected from the exterior. In the radical cure of hernia, especially inguinal, it is difficult to prevent the exposure of the wound, and I should despair of doing so without the aid of a very well fitted and securely fixed outside dressing. It would be easy to adduce a number of inguinal and scrotal wounds in which the outside dressing had prevented the wound from being soaked with urine.

After laparotomy another danger has to be guarded against. Owing to the anaesthetic and the nature of the operation vomiting is a frequent complication. During vomiting the contractions of the abdominal muscles are extraordinarily violent and special precautions are needed to prevent the access of air to the wound, and hence an accurately-fitted and fastened outside dressing becomes a wise precaution. In hot weather it can if necessary be easily dispensed with after the first forty-eight hours. But nowadays it is unusual to hear any complaints of discomfort, and the skin is hardly ever found reddened, much less blistered. 'For similar reasons an cutside dressing is of use in amputations of the breast and about the root of the neck. During vomiting, coughing, and breathing there is a strong probability of the entrance of air into the wound unless special precautions in the shape of an outside dressing be taken, Those which $I$ use are all cut to pattern and carefully fitted and fastened with straps and buckles. They are afterwards secured with bandages which are sewn on or reinforced with strapping.

The Influence of Environment.

It is probable that much has to be learnt about the influence of environment upon the results of operations. The term environment is rather loose and ambiguous, but we all have a general notion that it comprises the surroundings of the patient before, during, and after the operation. The surroundings during the operation have already been discussed, and likewise some of those which are requisite before and after the operation.

In this metropolis surgeons operate in public hospitals, private hospitals or nursing homes, and in private dwellings. It is exceedingly difficult to ascertain how far these three different environments influence the results as tested clinically by the mode of healing. I possess, however, a smal amount of information which will now be placed before you. The series begins March, 1900, and ends July, 1903.

I do not intend to weary you with a recital of the precautions which are taken in the organization of operations in private houses. Briefly the objects aimed at are accuracy and simplicity. Accuracy is attained by close personal supervision. I suspect that in order of danger come sponges, ligatures, and instruments, although the hands of the surgeon and of his assistants and the patient's skin are almost as dangerous. Now, every one of these is, and ought to be under the immediate control of the operator. The utensils and towels may be left to others, and are easily sterilized with heat. But it is always advisable to make precise inquiry into the manner of their disinfection before allowing them to be used. Care is likewise taken to see that the room in which the operation is performed is light, clean, and airy, and that the sanitation is perfect. The room is cleared out and prepared the day before and its air left to settle.

We may begin with fifty.eight wounds inflicted in private houses, hotels, or flats. As regards severity these are not all comparable to those in St. Bartholomew's Hospital or in nursing homes. A very critical operation has usually, in case of some sudden emergency arising, to be done in a nursing home close at hand. The list includes cases of amputation of the breast, amputation of the breast with removal of the pectoral muscles and axillary lymphatics, radical cure of hernia, arthrotomy, appendectomy, laminectomy, one of ligature of superficial femoral artery, removal of tumours, and so forth. I have omitted from this list cases in which pus was present and wounds communicating with septic gall bladders or the urinary bladder.

A case which died of shock after a long and difficult operation for the removal of a broad ligament tumour has been omitted, and also a death from shock after amputation of the thigh for diabetic gangrene. In malignant disease of the breast the ultimate safety of the patient often necessitates the removal of very large areas of skin. I am accustomed to close the whole of the huge wound by turning up long flaps of skin from the flank, or abdomen, or chest. When the wound is apparently aseptic these flaps preserve their vitality in a very remarkable manner, but the pointed tip sometimes dies and a small part of the wound has to heal by granulution. Under these circumstances it would be impossible to include the wound amongst the aseptic, although nearly the whole had healed by first intention, and the temperature and pulse had fallen to normal after the shock and reaction had passed away. In three breast amputations some part of the flap necrosed, and a portion of the wound had to heal by granulation.

Of the remaining fifty-five, one had slight haemorrhage and haematoma after a very severe operation for the removal of a malignant mass from beneath the clavicle. The growth was adherent to the subclavian vein, so that part of that vessel had to be excised. The patient made a good recovery, and, I am informed, without suppuration. Arthrotomy was performed upon a young lady for the exploration of the knee, with removal of a slipping internal semilunar fibro-cartilage. After the operation the course of events was uneventful until the fifth day, when the temperature rose, the fauces became inflamed, and a red skin eruption appeared. The knee then became more painful, and some blood-stained fluid was 
removed with an aspirator. This was examined by my colleague, Dr. F. W. Andrewes, and pronounced to be sterile. A small drainage tube was inserted, and slight suppuration occurred along its track and in the superficial part of the original wound. There followed a slight outbreak of eczema in the head and limbs which prevented the use of antiseptics other than lead and spirit lotion. Although this case was attended with great anxiety, the joint escaped without damage, and now possesses its full range of movement.

This is the only one out of numerous arthrotomies which has given me anxiety. The sanitation of the house was modern and seemed to be without a flaw, and the suppuration may have been, and probably was, caused by infection from the skin, for the patient was eczematous; but, on the other hand, the high temperature, the inflammation of the fauces, and the cutaneous eruption seemed to indicate the onset of one of the exanthemata. One would like to offer this to you as an explanation of the septic infection, but candour compels me to say that I have seen attacks of measles and of chicken-pox produce no ill-effects upon operation wounds. I can believe that sepsis might be conveyed by the blood stream from the ulcerated fauces of scarlet fever or diphtheria, but not in cases devoid of ulceration or suppuration. The rest of the cases healed by first intention. This gives a proportion of one case of suppuration and one of healing by granulation in fifty-five, or, including both, 3.63 per cent. of sepsis.

In London and its suburbs small private hospitals or nursing homes have multiplied in a remarkable manner. As yet, so far as I am aware, none has been built for the actual purposes of a hospital. All those with which I am acquainted have been started in private houses which have been altered and adapted to serve the new purpose. That they serve the purpose exceedingly well will be acknowledged on every hand, and those in charge having been, as a rule, nurses or sisters at large hospitals, are thoroughly acquainted with and devoted to their duties. A new building planned for the special purposes of a private hospital would probably be quieter and more convenient, but could hardly possess better operating theatres, or better roqms and appliances than are now available in these nursing homes.

The operations performed in nursing homes are not strictly comparable to those performed in private houses. Amongst them were some of great severity, and therefore requiring to be within, range of prompt attention. The operations included radical cure of hernia, appendectomy, ovariotomy, amputation of the breast, amputation of the breast with removal of pectoral muscles and of the axillary glands, nephrectomy, nephrorraphy, excision of varicose veins, laparotomy, and so forth. Two of the patients had double operations performed on them.

Eighty-two out of ninety-two wounds had healed by first intention when the patients left the nursing home. The wound made for the removal of an exostosis of the tibia was slightly pulled apart by premature walking, Twice after removal of the broast, pectoral muscles, and axillary lymphatics, a very small portion of the edge of the skin necrosed so that a small part healed by granulation, the rest per primam. Seven wounds were septic, about 7.6 per cent. So far as I can tell the causes of sepsis were as follows :

r.. After castration the dressing was disturbed, with exposure of the upper part of the wound ; there was slight suppuration along the course of the drainage tube, but the' silk sutures upon the spermatic cord and in the aponeurosis of the external oblique did not come out, and the rest of the wound healed by first intention. The inguinal canal had to be opened because a malignant growth had spread up the spermatic cord. The prevention of such an accident as the exposure of the wound ought not to be a difficult problem to either the surgeon or the nurse.

2. Laparotomy was performed to ascertain the nature of a tumour near the greater curve of the stomach. It proved to be a carcinoma involving the transverse colon, great omentum, great curve of the stomach, and the anterior abdominal wall. Some of the adhesions had to be separated. Ten days after the operation the pulse became quicker, the temperature rose, and a little pus escaped from the lower end of the wound. After this a rapid recovery ensued. The pus had a faecal odour, and I thought that its formation may have been in some.way due to the separation of the adhesions. This is rendered likelier from the fact separation of the adhesions. This is rendered likelier from the fact
that nine months after, the wound had healed I was told that another abscess formed and became a faecal leak. This is one of the perils in intra-abdominal malignant disease.

3. The wound suppurated after the removal of a very adherent vermiform appendix. The appendix burst and a quantity of faecal contents escaped into the wound. The patient made a rapid recovery; as no burfed sutures had been inserted, As a rule an accident of this kind can be araide: i, byt, should it occur, it is better to drain the iliac fossa for the first tho or three days after the operation.
4. A bursal tumour was removed from beneath the gluteus maximus, the latter being separated in the course of its fibres. The wound seemed so dry that no drain was inserted. A huge haematoma formed and had to be drained. The blood which escaped was septic, the pulse-rate was slightly increased, and the temperature was raised. After the septic clot had escaped recovery was rapid and perfect.

5. A large lipoma was removed from the back. No drain was inserted. A haematoma formed and was removed. The wound healed in part by granulation.

6. Radical cure of inguinal hernia was performed on both sides, The right side healed by first intention. A small haematoma formed on the left, followed by very slight suppuration and the extrusion of one of the deep sutures.

7. Suppuration occurred under the following circumstances after the radical cure of inguinal hernia in a patient weighing 7 st. On the right side he had an enormous scrotal hernia, which was irreduciblc and. and liable to attacks of obstruction. It also prevented him from following his occupation and rendered his life a misery. The hernial sac contained a part of the transverse colon, nearly the whole of the great omentum, the caecum and vermiform appendix, most of the right colon, and some part of ileum. The right colon was adherent to the posterior wall of the sae and was difflcult to reduce. The great omentum was excised and found to weigh $2 \frac{3}{4} \mathrm{lb}$. The abdominal wall was'repaired with silk and fishing gut and a drain inserted into the wound, which was large and deep. Sext day the scrotum was distended and the right testicle was very tender and inflamed. When this had subsided the wound, which had apparently healed, became painful and some very fetid pus escaped. This was not followed by the loss of any of the buried sutures. I cannot help suspecting that the right colon may have been pierced by one of the deep sutures. The ultimate result of this formidable operation has been highly satisfactory.

You will have noted that I said that eighty-two patients left the nursing homes with their wounds healed; but I know that two of these afterwards each developed a small sinus, and lost some buried sutures. These cases have both been mentioned before, when speaking about the fate of buried silk.

In one a very small haematoma formed beneath the skin of an appendectomy wound. The blood was let out, and the wound healed. The patient returned home within three weeks, but, as I have just said, a small sinus formed, and some of the silk escaped. In the other case, also, of appendectomy, the disease was tuberculous, and although the wound healed, and remained healed for some time, nevertheless a small sinus with some fungating granulation tissues formed, and I believe some silk was extruded. In this case some of the silk ligatures were placed upon tissue which was tuberculous, and it is most doubtful whether this ought ever to be done, In future I shall use biniodide catgut for tuberculous tissues. Obviously, these operations performed in nursing homes do not compare favourably with those in private houses, but, as I have already said, they are not strictly comparable.

The hospital operations were performed in St. Bartholomew's. The wards of that institution are more than 150 years old. Efforts have at various times been made to improve them by building sanitary towers, by reflooring, and by heating and ventilating. But the effort to alter and modernize old hospital buildings is hopeless, and the St. Bártholomew's wards remain exceedingly inconvenient when judged by modern standards. But the time is approaching when they will be replaced by modern structures supplied with ample air-space, light, and ventilation. Out of 175 wounds inflicted in the hospital, 153 healed by first intention, ro had some suppuration, and 12 others had necrosis of some portion of a skin flap, so that a portion of the wound healed by granulation: also a laparotomy wound which had its sutures removed too soon. : Omitting these, the percentage of suppuration is approximately 6 .

Amongst the wounds which healed by first intention are included two amputations of the thigh for gangrene of the leg. The circumstances were rather unusual.

An elderly woman was afflicted with a large uterine fibroid, and her left leg threatened to become gangrenous. At the request of my colleague, Dr. Griffith, I performed hysterectomy. Although this operation was foliowed by a good recovery, gangrene of the leg progressed, and the thigh had to be amputated." The wound healed by first intention, but convalescence was slow, owing to a mental illness which superiened. The second case was that of a boy who had the knee flexed acutely after old arthritis. He came to have amputation performed, buts I thought it reasonable to see: whether, resection could not be done. The limb was straightened with extreme diffculty, and soon the foot became gangrenous, and amputation had to be performed.

Thus we have a case in which a hysterectomy wound healed perfectly in spite of the presence of a gangrenous limb, and two : wounds which healed perfectly after the removal of a gangrenous limb. The explanation is simple, The limbs: were dead, but not infective. The amputations were done befora the dead tissues were teming with bacteria 
It would be misleading to say that 5.84 pereant of suppuration had occurred without giving brief details of the ciases. Suppuration varies in quality as well as in quantity. Not a little also depends upon the selection of cases. I have only rejected operations in which pus, a septic sinus. or a septic cavity was present at the time of the operation. I venture to think that something is to be learnt from the brief details of these cases. They are as follows:

r. The elbow was resected for advanced tuberculous disease, with much destruction of the articular cartilages, and of : the humerus and ulna. It is believed that the whole of the tuberculous synovial memorane and bone was taken away. The case did exceedingly well. although a little pus was discharged from the wound. The patient had had tuberculous caries of the spine and a psoas abscess.

Cases of this kind raise the important question of the incidence of suppuration after operations for tuberculous disease. I believe that these cases would not be more liable than others to suppurate, provided that every vestige of the tuberculous disease had been removed, and that no tuberculous material had heen smeared over or rubbed into the freshlycut tissues. But in most instances it is impracticable to fulfil one or both of those requirements, and, therefore, the chances of suppuration are increased. Should suppuration sccur, as it did in this case of resection, its onset is insidious and unaccompanied by marked alterations in the pulse-rate or temperature. Clearly, a wound in which there is reason to suspect the presence of tuberculous infection must be treated with special precautions. And, first, I use a drainage tube more frequently than for other cases. Next, it is injudicious to use silk for ligatures or buried sutures; prepared catgut is safest. Should catgut become infected with tubercle it does not stay long in the wound. And, lastly, I believe in the virtues of iodoform as an application and as a dressing. A little finely powdered crystals of iodoform is rubbed into the wound, and an iodoform gauze dressing is applied. Iodoform has to be used with great discretion, and it might be injudicious to put it into the cavity of a psoas abscess, although it may be used with safety in smaller cavities or wounds.

2. Another tuberculous wound suppurated and healed by granulation. A number of tubcrculous glands, together with a large piece of tuberculous skin, were removed from a man's neck, the wound was brough together, but with a considerable strain upon the sutures, and in the end it tore apart and the thound had to heal by granulation.

3. The radical cure of femoral hernia was followed by slight suppura ion and the loss of a buried suture.

4. Radical cure of hernia was performed on both sides for a man with double inguinal hernia. The right side was a simple operation, and th wound healed by frst intention. The left side was much more difficult as the sac contained omentum which was adherent at its neck. After the adhesions had been separated and the omentum ligatured and removed some blood welled out of the abdomen. The abdominal wall was freely divided, and the omentum again secured, although it had ceased to bleed. The peritoneal muscular, aponeurotic, and cutaneou layers of the abdominal wall were fastened together with silk sutures. The whole operation lasted two hours and twenty minutes. Ultimately there was slght subacute suppuration on the right side and two deep sutures were lost. The things which predisposed to this sepsis were probably: $(a)$ The prolonged exposure to the atmosphere: $(b)$ the bleed ing ; (c) the insertion of silk sutures into the muscular stratum.

5 and 6. Laminectomy is not a frequent operation in $\mathrm{my}$ experience, but the small number that $I$ have attempted have been followed by a very unusual and disconcerting amount of sepsis.

A youth 3 fell from a height of $30 \mathrm{ft}$. upon his back, and became completely paralysed below the level of the seventh intercostal space. Three weeks after the accident the spines and laminae of the third, fourth and fifth dorsal vertebrae were removed, and an epidural haemorrhage was found. After being in the hospital for over a year he was discharged much improved, with partial return of sensation and voluntary control of the bladder and rectum, and spastic condition of the muscles of the lower extremities, all of which reacted normally, though weakly, to electric excitation. Such is the report of my colleague, Mr. Bailey. who was then surgical registrar, but he omits to say that when the dressings were removed there was slight suppuration at one or two of the skin sutures. Fortunately this did not spread, but I certainly felt very anxious when I saw it. I think it is much to be regretted that all cases of suppuration are not recorded in the statistical tables of all of our hospitals.

Laminectomy was also performed to try and find a tumour of the spinal cord. The theca of the cord was bulged at the point where the tumour was supposed to be, but when the theca was opened no tumour could be seen. The cord was displaced after division of nerve roots, but still no tumour was in sight. The operation was followed by slight temporary improvement, although there was some suppuration in the superficial part of the wound and at some of the stitch holes. This patient died a month later of ascending pyelo-nephritis and with a parotid abscess. The wound was healed except at one stitch hole. The theca was healed and there was no sepsis about or within it. After the spinal cord had been taken out and cut across a long narrow timour was found in the anterior fissure, and at the exact level at which my colleague, Dr. Lewis Jones, had diagnosed it

In the tirst case I doubt not that the skin of the back was the source of infection, as it may also have been in the second case. The skin of the back is exceedingly thick, almost the thickest in the whole body. Moreover, it abounds in large sebaceous glands and sweat glands. Owing to the recumbent position of the patient the wound and dressings become bathed in perspiration. In a recent case of laminectomy I did my best to avoid these pitfalls, and the wound healed by first intention in spite of the presence of a septi: bedsore over the sacrum.

$I \mathrm{~h}$ ive heard it said that these paralysed patients are more predisposed to sepsis than the ordinary run, but I doubt whether this be true as regards wounds. The idea probably arises from the liability of the paralysed to suffer from retention of urine, with subsequent infection of the bladder, ureter, and kidneys.

7. An operation for ventrifixation of the uterus was followed by sup puration, but, rather to my surprise, I was told that no deep sutures had come out. Before performing this operation I was aware that there had been severe sepsis amongst the cases operated upon in the same theatre and ward. This did not deter me from operating, because I was then firm in the belief that wounds only became infected by contact. and that the methods I employed were an efficient safeguard against that danger. I am beginning to attach more importance to environment.

8. The mammary gland, pectoral muscles, and axillary lymphatics were excised for carcinomi. A portion of the skin flap slonghed and there was slight suppuration beneath it. The patient was 67 years' old, and suffered from asthma and bronchitis. $\Lambda$ severe cough is a troublesome complication when the wound involves the chest wall; as the entrance of air is prevented with difficulty.

9 and ro. Double radical cure of inguinal hernia was performed upon a porter. Small haematomata formed on both sides and became septic. None of the deep stitches were extruded, and the wound speedily healed. Stitches from the wound infected culture media, but unfortunately no cultures were made at the time of operation.

It is rather difficult to classify some cases. For instance, in carcinoma of the lymphatic glands of the neck secondary to carcinoma of the tongue I have imitated Mr. Butlin, and begun by turning the whole of the skin from off the anterior triangle of the neck by a large flap.

In four consecutive cases a small portion of the flap died, and a part of the wound had to heal by granulation. Now it might be said that the death of a small portion of a flap under such circumstances was not a very great misfortune. But this, to my mind, is not the right way of thinking. The operation is not'a perfect success unless all the flap survives and all the wound heals bo first intention. I have tried turning the flap backwards with its attached base along the anterior edge of the sterno-mastoid muscle, and I have tried turning it forward with its base along the middle line of the neck. On the whole. I think it is best to turn the flap back. But, unfortunately, the skin of the neck is very thin, and it seems difficult to include the platysma and deep fascia in the thickness of the flap. But the survival of these long okin flaps is, as I have already remarked, not altogether a question of blood supply. When the wound is aseptic, long thin nonvascular flaps adhere and survive, whereas in the presence of infection they would inevitably have perished. The aseptic process is more akin to Thiersch's skin grafting than to any ordinary skin-flap operation.

In six cases of removal of the breast, pectoral muscles; and axillary lymphatics a small portion of the edge or tip of the extensive skin flap necrosed, and a very small part had to heal by granulation. Healing by granulation occurred in the superficial part of a laparotomy wound for gastro-enterostomy. $A$ hasty house-surgeon removed the sutures prematurely.

Mr. Scott, to whom I am much obliged, has quite independently tabulated the results of the operations which I did during his house-surgeoncy. It is interesting to note how his estimate accords with my own, provided that the case with a pre-existing septic sinus be omitted, and, be it noted, that one of Mr. Scott's patients had two suppurating wounds.

Total wounds, 436 ; healed by first intention, 29 (80.5 per cent.); remaining, 7 .

Of these 7 cases, 3 had some necrosis of flap without sepsis; I had necrosis of flap with sepsis, but in this case there was a septic sinus at the time of operation; $x$ suppurated with loss of sutures; and 2 suppurated without loss of sutures, or time, one of them being a case of double radical cure in which both sides suppurated. Mr. Scott estimates the suppuration at 8.3 per cent., including the case with pre-existing septic sinus.

Some of the operations. which were followed by suppuration took a very long time to perform. What influence has the duration of an operation upon the chances of infection? 
Assuming that the atmospheric bacteria are harmless (an assumption which cannot for one moment be granted), then there is no reason why the wound should be infected either at the beginning, the middle, or the end of the operation But a surgical operation is a bacteriological experiment, and what bacteriologist would expose his culture media to the atmosphere for a couple of hours, and during all that time keep stirring them up? To ensure success bacteriological experiments must be done quickly and quietly, and so must surgical operations.

I can recall a few operations which have taken more than ninety minutes, and they have been followed by a larger proportion of sepsis than those of shorter duration.

Of course, the long exposure to atmospheric infection is not the only reason for this sepsis. I have already mentioned a case of radical cure of hernia in a very stout subject. Owing to his depth of fat a very big incision was required, and during the operation the loose and oily fat became bruised and full of clotted blood. Another very stout patient had an inguinal hernia full of inflamed and adherent omentum. The haemorrhage from the omentium was very free in spite of chain sutures of stout silk. Ultimately the wound had to be enlarged. The operation lasted two hours and a quarter. The patient made good progress, and left the nursing home at the end of three weeks with the wound soundly healed. Afterwards some pus formed beneath the scar, and after it had been let out some of the buried silk separated. Here, again, the thick layer of subcutineous fat was bruised and infiltrated with clotted blood. As no drain was inserted, some blood may have collected in the depths of the wound.

Some wounds are easier than others to protect agains atmospheric infection. Most abdominal wounds are of this nature. In the removal of the mammary gland, pectoral muscles, and axillary lymphatics, it is easy to protect the greater part of the huge wound with layers of antiseptic gauze.

It is difficult to see what effect any change in environment could have had upon this series of cases. In them the escape of blood into the wound and tissues around was the usual precursor of sepsis. The human blood is a most troublesome fluid; I sometimes wonder why the vessels should be filled with so strange a mixture. I often ask-but have never yet been told why-our own blood should clot and cause our own tissues to inflame? How often we meet with a sequence of events such as this : Blood shed into a serous cavity, into a joint, or amongst the tissues; clotting of the blood, and then inflammation of the tissues in contact with the clot; and, perchance, infection of the clot followed by its conversion into matter; for, although the infection usually. comes from without it may come from within, being carried by the blood stream or by the lymphatics.

These phenomena are clearly displayed in cases of subcutaneous injury, such, for instance, as a fracture of the patella. Some would ascribe the inflammation which ensues to the breaking of the bone and the tearing of the tissues, but I should ascribe it mainly to the extravasation of the blood. And acting on this belief, I take the greatest pains when wiring the patella to remove every particle of clot. In the subcutaneous operations which have been devised for fractured patella the clot is, of course, not taken away. But this is a trifling objection to that method of operation compared with another, for subcutaneous methods do not take away the piece of dorsal aponeurosis which always falls between the fragments.

The inflammatory effects of extravasated blood are very marked when it is in contact with that sensitive membrane the peritoneum. Twice I have been requested to operate for appendicitis and found instead a haematosalpinx. To my mind the error was venial. At the operation I saw the area of inflamed, engorged, and swollen peritoneum around the clots; and clinically the pain, elevation of temperature, and acceleration of the pulse had all seemed to justify the diagnosis. I suppose a physiologist could tell us exactly which element in the bload clot excited the inflammation, which raised the temperature, and which accelerated the pulse.

I have on three occasions seen the omental vessels bleed into the abdoinen after radical cure of hernia. The signs of peritonitis were very marked, and once they were of the most septic type. . The infection may have been introduced at the operation, but the operator had had no other cases of peritonitis. I am sure that had no bleeding occurred the fatal case would not have been recorded. But when blood clot is close to the intestines it may be infected from their interior, the intestinal bacteria finding their way through the coats of the intestines when the latter are inflamed or infiltrated with blood.

Although I have not had to tell you of a single instance of general septic infection, or even a single one of progressive suppuration, I am not contented, and fervently hope that better results may in future be obtained.

NOTE AND REFERENCES.

1 Trans. Med. Soc. Lond., vol. Xiv, 189r, p. 40, et seq. 2 Ibid., vol. xix, p. 26 . fig. 2. ${ }^{3} \mathrm{t}_{\text {; }}$ Dort of Surgical Registrar, Statistical Tables of St. Bartholomew's Hospital, 002, p. 194. Wounds of usual character, and including radical cure of hernia, appendectomy, intestinal anastomosis, amputation of breast and thigh, excision of knee, and so forth.

\section{MEMORANDA: \\ MEDICAL, SURGICAL, OBSTETRICAL, THFRA- PEUTiCal, PATHological, Ero.}

POTATOES AND GLYCOSURIA.

I HAVE seen lately potatoes mentioned as being more wholesome than ordinary bread for diabetics.

A patient, aged 57, with slight alimentary glycosuria, thought potatoes did not suit him. I found that after a luncheon of chop and plenty of bread there was no sugar in the urine, but an hour after a chop and plenty of potatoes. there was a large amount of sugar (I did not estimate it quantitatively.)

Thinking it over lately I made an infusion of a little wellboiled mealy potato in warm distilled water, and to my surprise found a large amount of grape sugar both by the copper and the phenyl-hydrazin test. Therefore potatoes, though an excellent food, are not good for diabetics.

If I have the opportunity I will see how new potatoes (when they come in) suit my patient. stourbridge.

George Birt, M.B.Lond.

\section{A NEW METHOD FOR DETECTING PLAGUE BACILLI} IN THE BLOOD.

Ir has hitherto been held that plague bacilli only occur in the general circulation in few cases, or at most when the patient dying. This is an error which has arisen from faulty methods of examining the blood films; the bacilli can be found in all cases and at all stages, and a case can be diagnosed almost as easily as a case of malaria.

In working at malaria with Ross's method it struck me that the same method would be-as it has proved to be-as useful in filarial blood, and a happy inspiration led me to examine a case of plague in the same manner, and, to my surprise, numbers of bacilli were easily found.

We-that is myself and Dr. Laing, assistant superintendent - have now examined numbers of cases, mostly on the first or second day of illness, and in every case the result has been positive. It is only necessary to make the film a little thinner and a little more spread out than in working at malaria to obtain good results.

Superintendent, Government Civil Hospital, Hong Kong.

THE TREATMENT OF HAEMOGLOBINURIC FEVER.

THE treatment of haemoglobinuric fever in British Central Africa has occupied the attention of Protectorate medical officers since 1895. Owing to lack of experience of the disease no particular line of treatment was at first followed, and such cases as were not treated with small doses of quinine were treated symptomatically.

In 1895 and the year following several cases came under observation, both at Zomba and Fort Johnston, and about this time Dr. Steudel, of the German Imperial Government, published a paper in which he strongly advocated the administration of large doses of quinine in the treatment of this disease-about $60 \mathrm{gr}$. per diem ; supporting his recommendation of these large doses by quoting a remarkable number of recoveries in his experience on the East Coast.

In the absence of any guide to treatment his method was immediately adopted in this Protectorate, and the mortality from haemoglobinuric fever during the years 1896,1897 , and 1898, was estimated at about 33 per cent. The cases which then presented themselves appeared to be of a severe type, and death from suppression of urine was. the termination in the majority of the fatal cases.

Early in 1899, and after an experience of several cases of 